\title{
Изменения фотоэлектрических свойств нелегированных пленок аморфного гидрированного кремния под влиянием предварительного освещения при повышенных температурах
}

\author{
(C) Н.Н. Ормонт, И.А. Курова \\ Московский государственный университет им. М.В. Ломоносова (физический фракультет), \\ 119991 Москва, Россия \\ E-mail: ormont@phys.msu.ru
}

Поступила в Редакцию 10 декабря 2019 г.

В окончательной редакции 16 декабря 2019 г.

Принята к публикации 16 декабря 2019 г.

\begin{abstract}
Проведено исследование влияния предварительной слабой засветки при повышенных температурах на фотоэлектрические свойства нелегированных пленок $\alpha$-Si:H. Установлено, что темновая проводимость и фотопроводимость пленок растут с увеличением интенсивности предварительной засветки, а параметр $\gamma$, определяющий зависимость фотопроводимости от интенсивности освещения, уменьшается вследствие увеличения доли бимолекулярной рекомбинации электронов на энергетических уровнях хвоста плотности состояний зоны проводимости. Предположено, что это может быть обусловлено наличием неконтролируемой примеси кислорода и увеличением электрически активной концентрации кислорода в результате предварительного освещения пенки при повышенных температурах.
\end{abstract}

Ключевые слова: аморфный гидрированный кремний, $\alpha$-Si:H, фотоиндуцированные метастабильные состояния, аномальный эффект Стеблера-Вронского, люксамперная характеристика, энергия активации, кислород.

DOI: 10.21883/FTP.2020.04.49142.9331

\section{1. Введение}

Фотоиндуцированное уменьшение темновой проводимости $\left(\sigma_{d}\right)$ и фотопроводимости $\left(\sigma_{\mathrm{ph}}\right)$ нелегированных пленок аморфного гидрированного кремния $\alpha$-Si:H под влиянием освещения при комнатных температурах (эффект Стеблера-Вронского - ЭСВ) [1] имеет научный и практический интерес и широко исследуется уже более 40 лет. Но исчерпывающего понимания механизмов этого эффекта в $\alpha$ - $\mathrm{Si}: \mathrm{H}$ до настоящего времени нет. Это связано, в частности, с тем, что пленки $\alpha-\mathrm{Si}: \mathrm{H}$ содержат большую концентрацию водорода, образующего различные связи с атомами кремния. Поэтому фотоиндуцированные изменения свойств $\alpha-\mathrm{Si}: \mathrm{H}$ могут определяться различными структурными перестройками этих водородных связей, с образованием разных дефектов, зависящих от микроструктуры исследуемой пленки.

Нами был обнаружен аномальный эффект СтеблераВронского - увеличение темновой проводимости и фотопроводимости нелегированных пленок $\alpha$-Si:H под влиянием освещения при повышенных температурах $(T>380 \mathrm{~K})[2-4]$. Ранее аномальный ЭСВ наблюдался и исследовался в легированных фосфором или бором и компенсированных пленках $\alpha$-Si:H после их освещения при повышенных температурах и объяснялся увеличением степени легирования пленок легирующими примесями [5-7].

В наших работах было предположено, что аномальный ЭСВ и другие фотоиндуцированные аномальные эффек- ты в нелегированных пленках $\alpha$-Si:H, предварительно освещенных при повышенных температурах, связаны с образованием двух типов метастабильных фотоиндуцированных дефектов — „быстрых“ и „медленных“, чьи энергетические уровни расположены соответственно в нижней и верхней половине запрещенной зоны. Быстрыми дефектами являются фотоиндуцированные оборванные связи кремния (ОС), которые определяют уменьшение темновой проводимости и фотопроводимости пленок, освещенных в области комнатных температур [8]. Нами было сделано предположение, что „медленные“ фотоиндуцированные метастабильные дефекты - это дефекты типа центровой водородной связи (ОС вблизи H-Si-связи) [2]. Образование центровых водородных связей в результате освещения пленок $\alpha$-Si:H при повышенных температурах предполагалось в ряде работ [9-12]. Отметим также, что образование энергетических уровней в верхней половине запрещенной зоны в нелегированных пленках $\alpha$-Si:H было обнаружено в работах $[9,13,14]$, но природа их не обсуждалась. В работе [15] было указано также, что центровая связь водорода в кристаллическом кремнии образует энергетический уровень донорного типа, расположенный в запрещенной зоне на 0.2 эВ ниже зоны проводимости.

В данной работе представлены результаты исследования изменений свойств нелегированных пленок $\mathrm{Si}: \mathrm{H}$ под влиянием их освещения при повышенных температурах, а также обсуждение механизмов этих фотоиндуцированных изменений. 


\section{2. Образцы и методика измерений}

Исследовались отожженные нелегированные пленки $\alpha-\mathrm{Si}: \mathrm{H}$ и эти же пленки после их изохронной засветки белым светом с разной интенсивностью $Q$ при температурах выше комнатных $\left(T_{c}>410 \mathrm{~K}\right)$. Пленки толщиной 0.8 мкм были выращены методом плазмохимического осаждения при температуре подложки $T_{S}=250^{\circ} \mathrm{C}$. Для сохранения новой структуры и свойств засвеченных при повышенной температуре пленок сразу после выключения засветки пленки быстро охлаждались до температур ниже комнатных и только затем при медленном нагреве измерялась температурная зависимость темновой проводимости пленок.

Измерения зависимости фотопроводимости $\sigma_{\mathrm{ph}}$ от интенсивности освещения $W$ исследуемых пленок $\alpha-\mathrm{Si}: \mathrm{H}$ (люксамперные характеристики - ЛАХ) проводились при температуре засветки пленки и выполнялись в цикле увеличения, а затем уменьшения интенсивности света, что было проверкой отсутствия изменения свойств во время измерения ЛАХ. Отметим, что все результаты измерений проверялись на воспроизводимость.

Для проведения исследований фотоиндуцированных изменений свойств этих пленок использовались криостат, обеспечивающий измерения в диапазоне температур от $T=100$ до $480 \mathrm{~K}$, и галогеновая лампа с ИК-фильтром и набором дополнительных нейтральных фильтров.

\section{3. Результаты исследований и их обсуждение}

На рис. 1 представлены температурные зависимости темновой проводимости $\sigma_{d}(1)$ и фотопроводимости $\sigma_{\mathrm{ph}}$ при различных интенсивностях света $(2,3,4)$ одной из исследованных нелегированных пленок $\alpha$-Si:H в отожженном состоянии. Видно, что приведенные величины и зависимости $\sigma_{d}$ и $\sigma_{\mathrm{ph}}$ характерны для нелегированных отожженных пленок $\alpha$-Si:H: экспоненциальная зависимость $\sigma_{d}(T)$ от обратной температуры и наличие ясно выраженного температурного гашения фотопроводимости $\sigma_{\text {ph }}$ при малых интенсивностях освещения. Из приведенного рисунка видно, что исследуемая пленка имеет высокую фоточувствительность. Ее величина, определяемая как отношение величин $\sigma_{\mathrm{ph}}$ к $\sigma_{d}$, при комнатной температуре и интенсивности освещения $W=50 \mathrm{MBT} \cdot \mathrm{cm}^{-2}$ имеет значение $3 \cdot 10^{5}$.

На рис. 2 и 3 приведена кинетика изменений темновой проводимости $\sigma_{d}(t)$, нормированной к ее равновесному значению при данной температуре, и фотопроводимости $\sigma_{\mathrm{ph}}(t)$ отожженной пленки $\alpha$-Si:H во время освещения светом с интенсивностью $W=50 \mathrm{MB} \cdot \mathrm{cm}^{-2}$ и при разных температурах. Видно, что при температурах вблизи комнатной в измеряемом промежутке времени наблюдается уменьшение $\sigma_{\mathrm{ph}}(t)$ и $\sigma_{d}(t)$ со временем освещения (эффект Стеблера-Вронского), при повышенных температурах — их увеличение (аномальный эффект

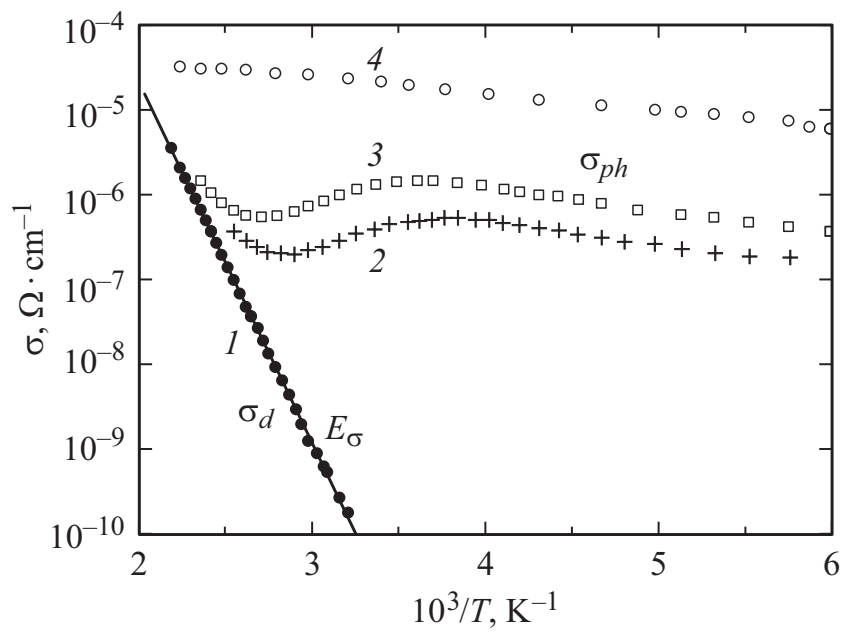

Рис. 1. Типичная зависимость темновой проводимости $\sigma_{d}(1)$ и фотопроводимости $\sigma_{\mathrm{ph}}$ при разных интенсивностях света $(2,3,4)$ от обратной температуры для одной из исследованных в данной работе нелегированных пленок $\alpha$-Si:H в отожженном состоянии. Интенсивности освещения пленки $W$ : $0.2(2), 0.6(3), 50 \mathrm{MBT} \cdot \mathrm{cm}^{-2}(4)$. Энергия активации темновой проводимости $E_{\sigma}=0.85$ эВ. Фоточувствительность пленки $k=\frac{\sigma_{\mathrm{ph}}}{\sigma_{d}}=3 \cdot 10^{5}$ при комнатной температуре и интенсивности освещения $W=50 \mathrm{MBT} \cdot \mathrm{cm}^{-2}$.

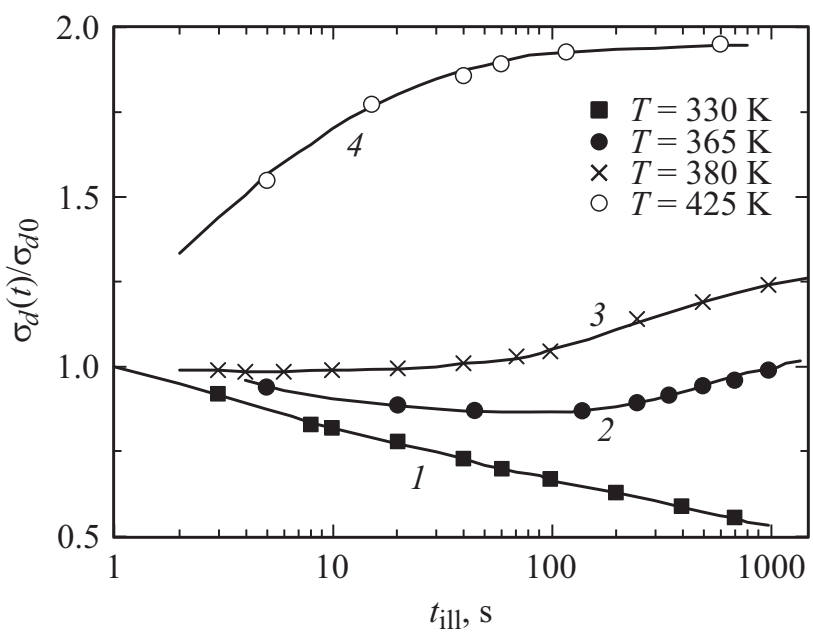

Рис. 2. Кинетика темновой проводимости отожженной нелегированной пленки $\alpha$ - $\mathrm{Si}: \mathrm{H}$ (образец 1), нормированной к равновесному значению $\frac{\sigma_{d}(t)}{\sigma_{d 0}}$, под воздействием освещения при разных температурах $(1,2,3,4)$. Интенсивность освещения пленки $W=50 \mathrm{мBT} \cdot \mathrm{cm}^{-2} \cdot \sigma_{d 0}-$ величина равновесной темновой проводимости при соответствующей температуре.

Стеблера-Вронского). При промежуточных температурах наблюдается суперпозиция двух процессов - быстрого уменьшения и медленного роста фотопроводимости и темновой проводимости. Скорости этих процессов различаются и растут с температурой.

На рис. 4 приведены температурные зависимости темновой проводимости отожженной пленки $\alpha-\mathrm{Si}: \mathrm{H}(1)$ и той же пленки после предварительной изохронной 


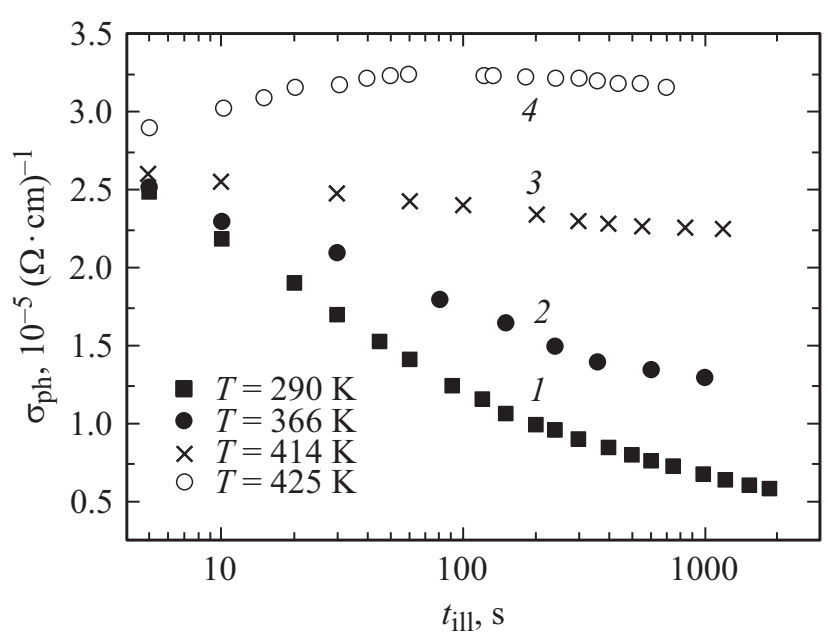

Рис. 3. Кинетика фотопроводимости $\sigma_{\mathrm{ph}}(t)$ отожженной нелегированной пленки $\alpha-\mathrm{Si}: \mathrm{H}$ (образец 1 ) под воздействием освещения при разных температурах. Интенсивность освещения пленки $W=50 \mathrm{MBT} \cdot \mathrm{cm}^{-2}$.

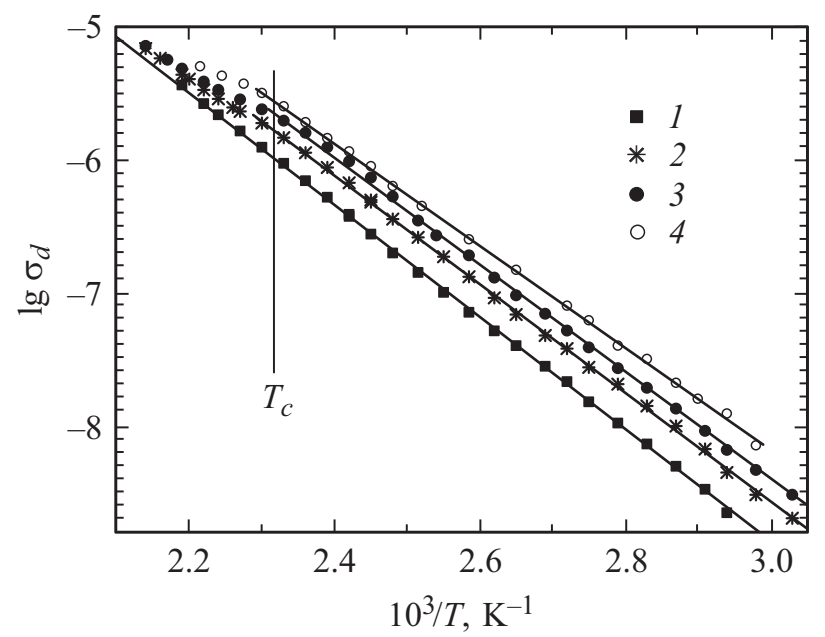

Рис. 4. Температурная зависимость темновой проводимости $\sigma_{d}$ отожженной пленки $\alpha$-Si:H (образец 1) и предварительно изохронно засвеченной этой же пленки при разных интенсивностях света $Q$ (образцы 2,3,4) в течение 10 мин и при температуре $T_{c}=425 \mathrm{~K}$. Номера кривых совпадают с номерами образцов. Проводимость пленки измерялась при медленном нагреве после быстрого охлаждения от $T=425 \mathrm{~K}$. Интенсивности предварительной засветки пленки: 0.6 (образец 2), 15 (образец 3) и $50 \mathrm{MBT} \cdot \mathrm{cm}^{-2}$ (образец 4).

засветки в течение 10 мин, с разной интенсивностью света $Q: 0.6(2), 15(3)$ и $50 \mathrm{MBT} \cdot \mathrm{cm}^{-2}(4)$ при температуре $T_{c}=425 \mathrm{~K}$. Все зависимости $\sigma_{d}(T)$ измерялись при повышении температуры сразу после выключения засветки и быстрого охлаждения пленок до низких температур.

Видно, что предварительно засвеченные пленки имеют более высокую величину темновой проводимости $\sigma_{d}$ и при $T<T_{c}$, где $T_{c}$ - температура засветки пленки, $\sigma_{d}$ экспоненциально зависит от обратной температуры. При увеличении температуры $T$ выше $T_{c}$ наблюдается отклонение темновой проводимости засвеченной пленки $\sigma_{d}$ от экспоненциальной зависимости и приближение ее к равновесной величине $\sigma_{d}(T)$ для отожженной неосвещенной пленки. Это указывает на термическую релаксацию фотоиндуцированной темновой проводимости засвеченных пленок. Энергия активации экспоненциальной зависимости $E_{\sigma}$ уменьшается от величины 0.85 эВ для отожженной пленки до 0.77 эВ при увеличении интенсивности предварительной изохронной засветки пленки $Q$.

Установленные фотоиндуцированные изменения величины и температурной зависимости темновой проводимости засвеченных пленок могут быть связаны с увеличением энергии Ферми, обусловленным изменением распределения плотности электронных состояний в запрещенной зоне.

На рис. 5 приведены зависимости фотопроводимости $\sigma_{\mathrm{ph}}$ от интенсивности освещения $W$, т. е. люксамперные характеристики (ЛАХ) отожженной (1) и предварительно засвеченных $(2-4)$ пленок $\alpha$-Si:H при $T_{c}=425 \mathrm{~K}$ в области малых значений $W$. Видно, что величина фотопроводимости всех предварительно засвеченных пленок $\sigma_{\mathrm{ph}}$ больше, чем у отожженной пленки, и возрастает с увеличением интенсивности предварительной засветки $Q$.

Зависимость фотопроводимости $\sigma_{\mathrm{ph}}$ от интенсивности освещения $W$ для всех исследованных пленок различна. Это различие характеризуется параметром $\gamma=\frac{d\left(\log \sigma_{\mathrm{ph}}\right)}{d(\log W)}$. Из наших измерений ЛАХ было получено, что параметр $\gamma$ для исследованных нами пленок $\alpha$-Si:H изменяется от значения, близкого к единице для отожженной пленки, до величии $\sim 0.65$ при увеличении интенсивности предварительной засветки пленки $Q$.

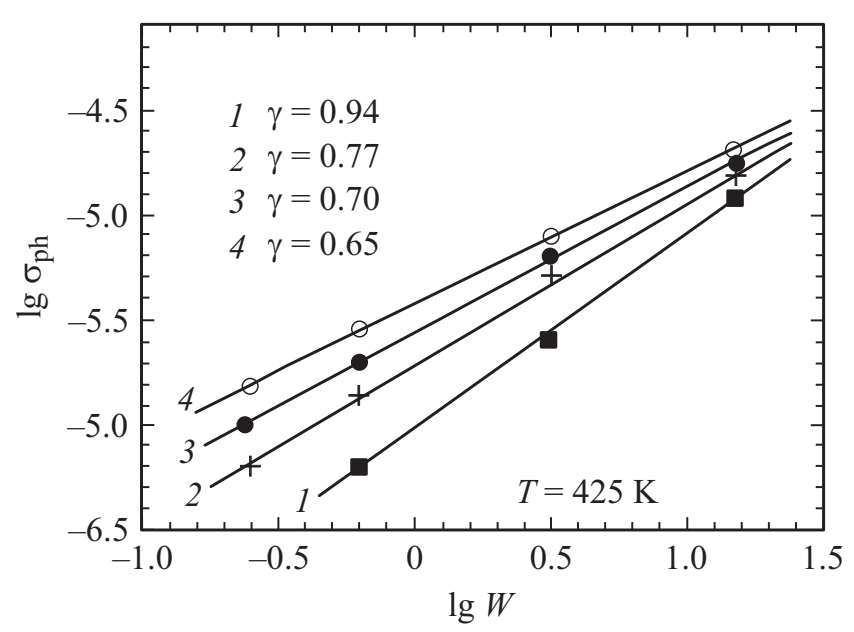

Рис. 5. Зависимость фотопроводимости $\sigma_{\text {ph }}$ от интенсивности света $W$ (ЛАХ) при $T=425 \mathrm{~K}$ для отожженной $(1)$ и предварительно изохронно засвеченной с разной интенсивностью $Q$ той же нелегированной пленки $\alpha-\mathrm{Si}: \mathrm{H}(2,3,4)$ при $T_{c}=425 \mathrm{~K}$. Номера кривых совпадают с номерами образцов. Время предварительной засветки - 10 мин при температуре $425 \mathrm{~K}$. Интенсивности предварительной засветки пленки: 0.65 (2), $15(3)$ и $50 \mathrm{MBT} \cdot \mathrm{cm}^{-2}(4)$. Параметр $\gamma=\frac{d\left(\log \sigma_{\mathrm{ph}}\right)}{d(\log W)}$ составляет: 0.94 (1), 0.77 (2), 0.70 (3), 0.65 (4). 
Таким образом, проведенные исследования показали, что в результате предварительной засветки нелегированных пленок при повышенной температуре происходят изменения ряда фотоэлектрических параметров: $\sigma_{d}, E_{\sigma}$, $\left(E_{C}-F_{0}\right), \sigma_{\mathrm{ph}}$ и $\gamma$. Эти изменения сохраняются при выключении предварительного освещения после быстрого охлаждения пленки для всех $T<T_{c}$.

Ранее подобные изменения фотоэлектрических параметров наблюдались в засвеченных при повышенных температурах пленках $\alpha$-Si:H $n$-типа, легированных фосфором и бором [5]. Была предложена модель, объясняющая эти изменения. Модель была основана на предположении об увеличении степени легирования пленок примесями и, следовательно, увеличении энергии Ферми в них при освещении пленок при повышенных температурах. Это приводит к увеличению заполнения электронами хвоста плотности состояний зоны проводимости и, следовательно, к увеличению доли бимолекулярной рекомбинации, что уменьшает величину параметра $\gamma$.

Данная модель может быть применима для объяснения наблюдаемых аналогичных фотоиндуцированных изменений параметров наших предварительно засвеченных нелегированных пленок при предположении присутствия в них неконтролируемой примеси. Исследованные нами нелегированные пленки характеризуются малой величиной темновой проводимости при комнатной температуре и большой величиной ее термической энергии активации, Это может быть связано с наличием компенсирующей неконтролируемой примеси. Неконтролируемой примесью, часто присутствующей в пленках $\alpha-\mathrm{Si}: \mathrm{H}$, является кислород. Кислород как многозарядная примесь может быть в $\alpha$-Si:H как донором, так и акцептором, образующим энергетические уровни в нижней и верхней половинах запрещенной зоны $\alpha$-Si:H [13]. Можно предположить, что при комнатных температуpax и малой концентрации кислорода по сравнению с концентрацией оборванных связей в наших образцах имеется донорный уровень кислорода, расположенный в нижней половине запрещенной зоны. При освещении пленки при повышенной температуре происходит увеличение концентрации электрически активного кислорода с образованием в верхней половине запрещенной зоны акцепторного уровня кислорода. Вследствие этого повышается энергия Ферми и увеличиваются темновая проводимость и фотопроводимость пленки, которые растут с увеличением интенсивности ее изохронной предварительной засветки. При этом также увеличивается доля бимолекулярной рекомбинации электронов на уровнях хвоста зоны проводимости, что уменьшает параметр $\gamma$.

\section{4. Заключение}

Установлено, что под влиянием предварительной засветки нелегированных пленок $\alpha$-Si:H при $T=425 \mathrm{~K}$ увеличиваются их темновая проводимость и фотопроводимость и уменьшается параметр $\gamma$, определяющий зависимость фотопроводимости от интенсивности освещения.
Предположено, что эти эффекты могут быть обусловлены наличием неконтролируемой примеси кислорода в пленках и увеличением в них концентрации электрически активного кислорода в результате освещения при повышенных температурах.

\section{Конфликт интересов}

Авторы заявляют, что у них нет конфликта интересов.

\section{Список литературы}

[1] D.L. Stabler, C.R. Wronski. Appl. Phys. Lett., 31, 292 (1977).

[2] И.А. Курова, Н.Н. Ормонт. ФТП, 47 (6), 757 (2013).

[3] И.А. Курова, Н.Н. Ормонт. ФТП, 49 (5), 603 (2015).

[4] И.А. Курова, Н.Н. Ормонт. ФТП, 51 (4), 440 (2017).

[5] R. Bube, D. Redfield. J. Appl.Phys., 66 (7), 3074 (1989).

[6] J. Takada, H. Fritzsche. Phys. Rev. B, 36 (3), 1706 (1987)

[7] J. Yoon, K. Kim, J.Y. Park. J. Appl.Phys., 91 (12), 3074 (2002).

[8] N.F. Mott. Fil. Mag. B, 51, 177 (1985).

[9] C. Longeaud, D. Roy, O. Saadane. Phys. Rev. B, 65, 085206 (2002).

[10] K. Morigaki, H. Hikita. Phys. Status Solidi C, 8 (9), 2564 (2011).

[11] H. Fritzsche. Ann. Rev. Mater. Res., 31, 47 (2001).

[12] P. Kounavis. J. Appl. Phys., 97, 023707 (2005).

[13] I. Sakata, T. Kamei, M. Yamanaka. Phys. Rev. B, 76, 075206 (2007).

[14] I. Sakata, T. Kamei, M. Yamanaka. J. Non-Cryst. Sol., 358, 2048 (2012).

[15] B. Holm, K. Bonde Nielsen, B. Bech Nielsen. Phys. Rev. Lett., 66, 18 (1991).

Редактор Г.А. Оганесян

Modification of photoelectric properties of undoped hydrogenated amorphous silicon films under the influence of preliminary illumination at elevated temptrature

\author{
N.N. Ormont, I.A. Kurova \\ Lomonosov Moscow State University \\ (Faculty of Physics), \\ 119991 Moscow, Russia
}

\begin{abstract}
The effect of preliminary weak illumination at elevated temperatures on the photoelectric properties of undoped $\alpha-\mathrm{Si}: \mathrm{H}$ films is studied. It was found that the dark conductivity and photoconductivity of films increases with increasing intensity of preliminary illumination, and the parameter $\gamma$ determining the dependence of photoconductivity on the light intensity decreases due to an increase in the fraction of bimolecular recombination of electrons at the energy levels of the tail of the density of states of the conduction band. It is assumed that this may be due to the presence of an uncontrolled oxygen impurity and an increase in the electrically active concentration of oxygen as a result of preliminary illumination of the films at elevated temperatures.
\end{abstract}

